\title{
Designing Configurable Arm Rehabilitation Games: How Do Different Game Elements Affect User Motion Trajectories?
}

\author{
Arzu Guneysu Ozgur ${ }^{1}$, Maximilian J. Wessel ${ }^{2}$, Thibault Asselborn ${ }^{1}$, Jennifer K. Olsen ${ }^{1}$, Wafa Johal ${ }^{1,4}$, \\ Ayberk Özgür, Friedhelm C. Hummel ${ }^{2,3}$ and Pierre Dillenbourg ${ }^{1}$
}

\begin{abstract}
For successful rehabilitation of a patient after a stroke or traumatic brain injury, it is crucial that rehabilitation activities are motivating, provide feedback and have a high rate of repetitions. Advancements in recent technologies provide solutions to address these aspects where needed. Additionally, through the use of gamification, we are able to increase the motivation for participants. However, many of these systems require complex set-ups, which can be a big challenge when conducting rehabilitation in a home-based setting. To address the lack of simple rehabilitation tools for arm function for a home-based application, we previously developed a system, Cellulo for rehabilitation, that is comprised of paper-supported tangible robots that are orchestrated by applications deployed on consumer tablets. These components enable different features that allow for gamification, easy setup, portability, and scalability. To support the configuration of game elements to patients' level of motor skills and strategies, their motor trajectories need to be classified. In this paper, we investigate the classification of different motor trajectories and how game elements impact these in unimpaired, healthy participants. We show that the manipulation of certain game elements do have an impact on motor trajectories, which might indicate that it is possible to adapt the arm remediation of patients by configuring game elements. These results provide a first step towards providing adaptive rehabilitation based upon patients' measured trajectories.
\end{abstract}

\section{INTRODUCTION}

Success of rehabilitation after stroke or traumatic brain injury depends on three different factors: feedback, repetition and motivation [1]. With the advancement in computerbased technologies and interaction modalities in the last decades, new rehabilitation technologies are designed with these three factors in mind. In order to improve engagement and increase the intensity of exercise, new rehabilitation technologies commonly include game-like elements such as entertaining graphics, automated difficulty adaptation, and feedback mechanism [2], [3], [4].

Prominent examples for these systems include virtual environments, video capture systems, game consoles and robots. With these new technologies, motor rehabilitation

\footnotetext{
*This work is supported by the Swiss National Science Foundation through the NCCR, the Defitech and the Wyss Foundation.

${ }^{1}$ Arzu Guneysu Ozgur, Pierre Dillenbourg, Jennifer K. Olsen, Wafa Johal and Thibault Asselborn are with CHILI Lab, EPFL, Switzerland. name. surnamedepfl.ch

${ }^{2}$ Maximilian J. Wessel and Friedhelm C. Hummel are with, Defitech Chair of Clinical Neuroengineering, Center for Neuroprosthetics and Brain Mind Institute, EPFL, Switzerland. name.surname@epfl.ch

${ }^{3}$ Friedhelm C. Hummel is with Clinical Neuroscience, University of Geneva Medical School, Switzerland.

${ }^{4}$ Wafa Johal is with the BIOROB Lab at EPFL, Switzerland.
}

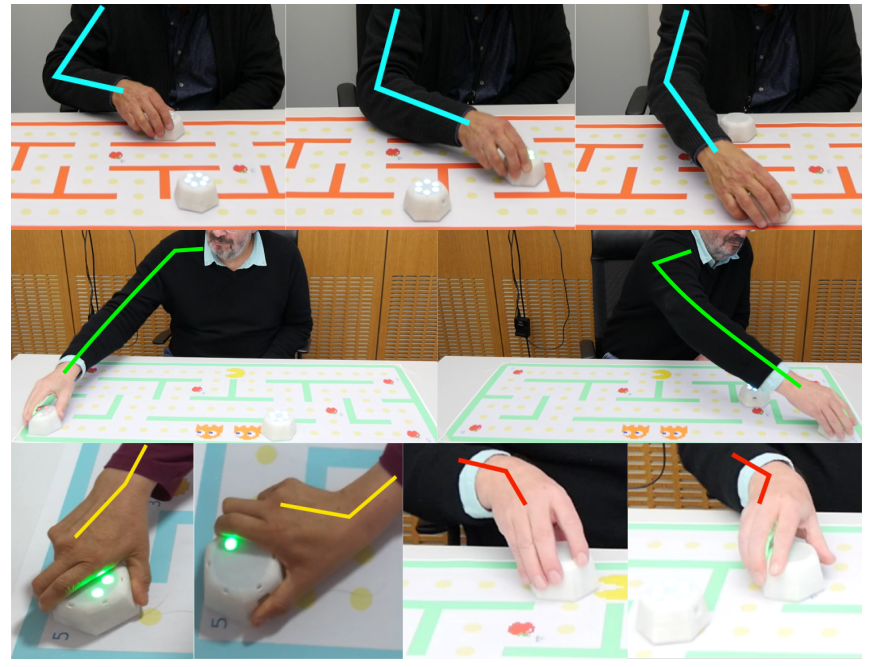

Fig. 1: Expected exercise activities during game motions [8].

can be made much more attractive for patients than conventional neuromotor rehabilitation, which is often repetitive and frustrating [5]; many previous studies agree that patient motivation can be significantly improved with the use of such new rehabilitation tools [5], [6], [3]. However, many of these tools do not address the challenges of home rehabilitation, such as the re-purposing of rooms, shared devices such as TV and computer and available space in the home [7], [8]. Current tools typically need a special room or an isolated space in the therapy center and home with a dedicated computer or TV, limiting the portability of the system.

To provide more compact and intuitive rehabilitation for home-therapy, we previously proposed a novel rehabilitation platform, Cellulo, composed of small-sized, graspable and haptic-enabled tangible robots. In a typical Cellulo game, patients move their designated tangible robot(s) while other robots perform their own, autonomous motions to affect the patients' motion. By augmenting the therapy with robotic technologies such as precise localization and locomotion, we hypothesized that various aspects of rehabilitation can be improved [8].

Taking into account the multi-faceted nature of stroke, and the evidence that not all patients benefit equally from rehabilitative treatments, it becomes clear that precision medicine strategies with the goal to tailor the interventions to the individual patient is promising. In the case of Cellulo, this can be done by shaping individual patients' specific motion trajectories performed during the game; which, if 
done according to the patient motion needs during the respective training sessions, would translate into more fitting rehabilitation exercises overall [9]. We anticipate that we can analyze the user motion trajectories in order to understand their level of motor skills and playing strategies, and use these to modulate our game elements to drive the user into the required trajectories.

This paper presents the evaluation of 33 unimpaired participants' motion trajectories while playing games on our rehabilitation platform with varying configurations. The goal of these evaluations is to determine how different configurable game elements affect the user's motion trajectory and to examine how user performance is related to these trajectories. We show that it is indeed possible to adapt the arm remediation of patients by configuring certain elements of our game, and lay the foundation for future adaptive gamified exercise platforms designed for participants with chronic upper limb impairment.

In the future, we aim to adapt these game elements to also encourage the patients to do the required arm motions. By learning from observations of the patient's trajectory, we aim to subsequently produce new patient-specific physiotherapeutic tasks and configuration suggestions.

\section{GAMIFIED REHABILITATION PLATFORM WITH TANGIBLE ROBOTS}

Our gamified rehabilitation platform consists of tangible Cellulo robots and different mazes on paper sheets designed with a theme close to the Pacman game [8]. Cellulo is a handheld, small-sized, mobile, haptic robot that operates on printed paper sheets. The platform provides fast and accurate localization of many such robots so we can deploy and log data to record all the interactions during the game. The activity itself is printed on paper sheets that can feature any desired graphical game elements defined as active zones.

The Cellulo for rehabilitation project aims to provide practical, easy to use and intuitive gamified upper-arm rehabilitation by using these tangible robots as game agents and objects. Our first game, Pacman, is designed iteratively with participation of stroke, brachial plexus and cerebral palsy patients (18 in total) and seven therapists in four different therapy centers [8].

A number of game elements are designed for tuning speed, accuracy, range of motion and challenge level; these are: (1) Different maps with various mazes or sizes, (2) One or two ghost(s) robot(s) chasing the Pacman, (3) Speed of the chasing robot(s) and (4) Optional rules: (4.a) Turn rule where the user can only collect the fruits by rotating the robot on top of them, (4.b) Cross border penalty rule where the user loses the last eaten fruit when he/she crashes into a wall, (4.c) Haptic feedback rule to provide haptic informative assistance when the user crashes into a wall [8].

Since the map is designed according to the possible range of motion of the human, the basic stroke rehabilitation exercise motions, namely elbow flexion and extension, shoulder abduction and adduction, grasping and wrist ulnar and radial deviation are expected during gameplay (see Fig. 1)

\section{ANALYSIS OF MOTION TRAJECTORIES}

We collected data from 33 unimpaired participants aged between 18 and 80. All participants took part in two sessions in our laboratory at EPFL Lausanne Campus or Geneva Campus Biotech after obtaining their informed written consent. Each participant played 52 games on three different maps within two consecutive days of experiments with changing game configurations and increasing difficulty per map.

There are six targets in the game that have been gamified as apples for the Pacman to eat. The user is expected to collect all six apples as quickly and precisely as possible to finish the game. In our game, precision is defined as not crashing into the maze walls. The ghost $\operatorname{robot}(\mathrm{s})$ chase(s) the user's robot during the game in order to catch it; all previously collected apples are lost if caught. The game then continues until six apples are eventually collected.

Since this data would be a baseline for rehabilitation purposes, we began with very easy games and increased the difficulty by introducing a new game element one at a time. Each participant played with three different maps with changing orders. These are the small yellow $(42 \mathrm{~cm} \times 62 \mathrm{~cm})$, large orange $(42 \mathrm{~cm} \mathrm{x} 96 \mathrm{~cm})$ and large green maps $(42 \mathrm{~cm} \mathrm{x}$ $96 \mathrm{~cm}$ ), which will henceforth be referred to as Map Y, Map O and Map G respectively.

Each participant played 27 games the first day ${ }^{1}$ and 26 games the second day, with 11 different configurations. The speed of the ghost is set to $20 \mathrm{~mm} / \mathrm{s}$ for the very first game of the each day, which is set to 40,60 and $60 \mathrm{~mm} / \mathrm{s}$ for the next three games. Next, haptic feedback is turned on and two more games with $60 \mathrm{~mm} / \mathrm{s}$ are played. After that, the penalty rule is introduced and two more games are played with 60 and $100 \mathrm{~mm} / \mathrm{s}$ ghost speed. Then, the turn rule is introduced and two more games with 60 and $100 \mathrm{~mm} / \mathrm{s}$ ghost speed. Afterwards, a second ghost is introduced and three more games are played with 60, 100 and $100 \mathrm{~mm} / \mathrm{s}$. Then, the map is changed and all above configurations (except 20 and $40 \mathrm{~mm} / \mathrm{s}$ ghost speed) are repeated with this second map. After this repetition, two extra games are played with the hardest configuration (penalty rule, $100 \mathrm{~mm} / \mathrm{s}$ speed, second ghost and turn rule).

For the goal of providing an adaptive game platform, our method is to use the trajectory data to estimate the user strategy, which will allow us to choose the appropriate counter strategy for the ghosts to make the game more adaptive to the level of the user. Game movements are timeseries of user's Pacman robot coordinates, recorded from the start of the each game until collecting the last apple. During the game, position $(x, y)$ and orientation of each robots are recorded with close to $100 \mathrm{~Hz}$ frequency and sub$\mathrm{mm}$ accuracy.

Dynamic Time Warping (DTW) is a well known distance method used in trajectory analysis in human activity monitoring [10]. This makes DTW particularly suitable for Pacman play data since it allows to measure the similarity between

\footnotetext{
${ }^{1}$ The first day was closed with an extra easy configuration game to measure any overnight learning effect, which is not discussed here.
} 

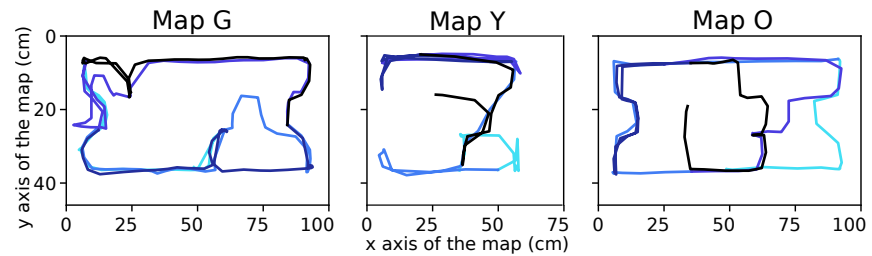

Fig. 2: Example game trajectories in blue colored clusters (light to dark denotes start to end).

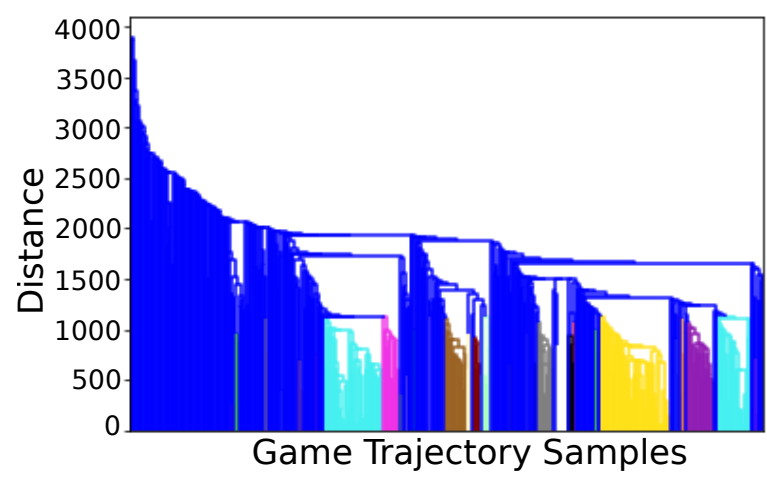

Fig. 3: Dendogram of clusters in Map Y.

temporal sequences without being affected by speed differences between participants, or by intervals when participants remained idle in one location.

For trajectory classification, namely the model construction for predicting the class labels of moving objects based on their trajectories, a number of methods have been reported in the literature. Many of these methods use the shapes of whole trajectories for classification, leading to limited classification capability when discriminative features appear at parts of trajectories or are not relevant to the shapes of trajectories [11]. For this reason, we choose hierarchical clustering [12] as it captures the lower-level trajectory-based features using movement patterns.

Pacman gameplay trajectories typically include paths containing several backward movements depending on the ghost behaviours and positions. During several gameplays, users learn shortest paths in the maps for collecting apples in an

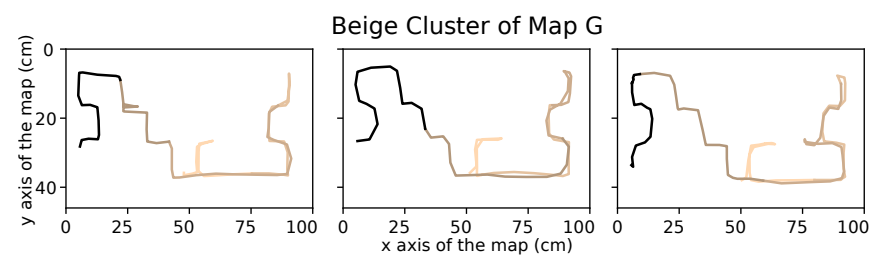

Fig. 4: Example game trajectories in Map G corresponding to 3 different participants (light to dark denotes start to end).

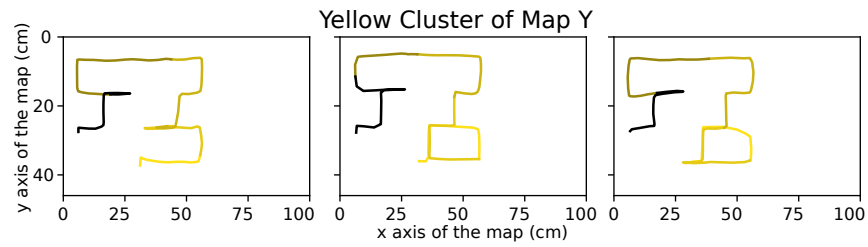

Fig. 5: Example game trajectories in Map Y corresponding to 3 different participants (light to dark denotes start to end). optimized manner and without being caught by the ghosts.

Since each map has different maze type and size, clustering is done per map. One example dendogram representing clusters of map Y can be seen in Fig. 3. It can be observed that hierarchical clustering provides some clusters with less distance difference such as yellow and purple clusters in Map $\mathrm{Y}$, which mostly corresponds to shorter paths on the maps. Furthermore, the large clusters marked with blue in each map correspond to paths having several backward motions, more directional changes within the maze and passes through the same maze parts several times. Trajectories within the blue cluster therefore include more motion. An example trajectory within the blue cluster of each map is shown in Fig. 2. Finally, some example trajectory paths of other clusters can be seen in Fig. 4 and Fig. 5. In these figures, the direction of the trajectory is indicated with increasingly darker colors.

\section{RESULTS}

\section{A. Relationship Between Trajectory and Performance}

Performance of a user in a given game is calculated using the total time to finish the game (collecting 6 apples) and the mean deviance from the middle of the path while moving along the maze, as follows: $1 /\left(T_{\text {total }} \times D_{\text {mean }}\right)$. These two metrics also take into account the time lost by making accuracy related errors, namely losing the last eaten apple due to the border crossing penalty rule, time lost by being caught by a ghost and the speed of the user. Fig. 6 shows the relationship between game performances and trajectory types for each map. Having longer paths, the blue cluster of each map has the one of the lowest performances as expected.

\section{B. Effect of Game Elements on Trajectory Distribution}

Several elements can be adapted within a game setting that will change the way users travel through the map (different strategies). This section will only focus on the effects of the number of ghosts, speed of the ghost(s) and map type on users' trajectories.

1) Effect of number of ghosts: Fig. 7 shows the effect of ghost number to cluster distributions for each map: The proportion of blue cluster increases in Map G and Map O with the addition of the second ghost, which could suggest that users have less defined and less shortest path strategies when the complexity of the game increases. Concerning Map $\mathrm{Y}$, the proportion of blue cluster remains similar since the small size of this map internally inhibits emergence of clear strategies, as the ghost can always be found near the user's position.

If the results are interpreted by focusing only on the other colors than blue, it can be observed that some dominant cluster sizes change with increasing number of ghosts. For instance, in Map G, dark-green and light purple clusters have the highest impact in 1 ghost condition, which are largely replaced by the yellow cluster in 2 ghosts condition. In order to build a deeper understanding of the reason behind such changes, visually examining some trajectories within these clusters becomes highly informative: Fig. 8 shows that the clusters of games having only one ghost involve 

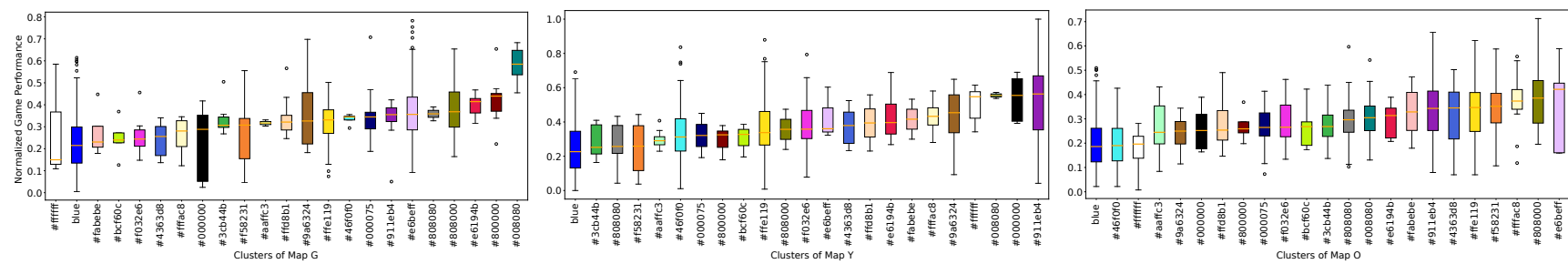

Fig. 6: Game performance v.s. clusters. We observe that the blue cluster has one of the lowest performances in each map, as expected.

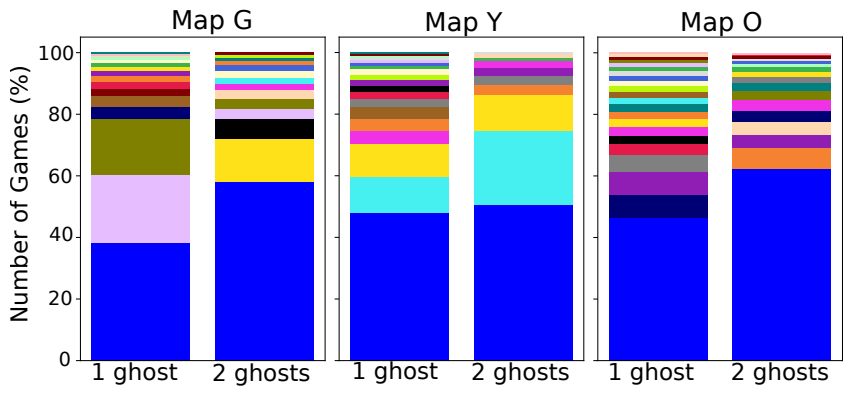

Fig. 7: Effect of number of ghosts on the cluster distribution. Each color represents a different cluster.
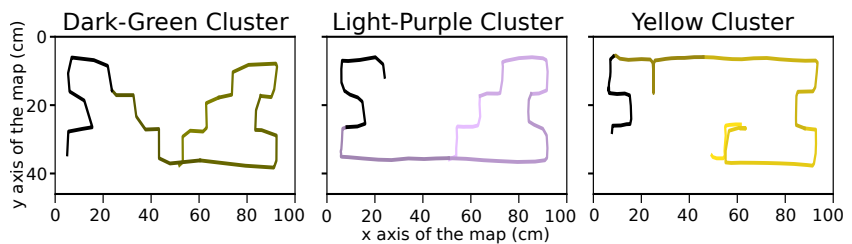

Fig. 8: Dark green, light purple and yellow cluster trajectories of Map G (light to dark denotes start to end).

trajectories where the user is travelling through the middle of the map vertically (see dark green, light purple). On the other hand, in the yellow cluster, users chose to travel around the map borders with more horizontal motions to be away from two ghosts chasing them. Similar horizontal movement tendencies are also seen in the clusters of other maps that have increased percentage with 2 ghosts condition. This shows that adding more ghost to the game can increase the probability of having wider shoulder motions.

This result suggests that in the future, looking more in detail into the nature of trajectory clusters may help us to extract further results in what the clusters mean in a kinematic or strategic sense, e.g. favouring change in direction, favoring easy v.s. difficult target to pick first etc.

2) Effect of speed increase: A number of game configuration transitions during the experiment induces ghost speed increase while other rules in the configuration stay the same. The effect of some of these transitions are shown in Fig. 9.

Fig. 9 left part shows the cluster distributions in 20, 40 and $60 \mathrm{~mm} / \mathrm{s}$ ghost speeds. $20 \mathrm{~mm} / \mathrm{s}$ belongs to the very first game of each day with a new map, which naturally results in higher exploration tendency, which can be seen as higher blue cluster percentage. As the learning of the map increases through time (while the ghost speed increases from 20 to 40 and then $60 \mathrm{~mm} / \mathrm{s}$ ), participants may naturally move towards clusters with shorter path trajectories. This effect is visible as generally decreasing blue cluster impact over time. However, since learning and speed increase are jointly affecting the clusters, it cannot clearly be said which of these two factors has the greater impact, or whether the speed increase has a significant effect in decreasing the blue cluster impact.

When the speed is increased while there are two ghosts in the game, the proportion of blue cluster in Map Y becomes approximately twice as much of the slower speed, showing more increase compared to the one ghost condition. This suggests that two ghosts with high speed in the small maze can push the user to move more and prevent winning the game with a short path.

We summarize our interpretations of the speed effect as follows: The speed increase may result in more motion if the speed is high enough to push the user to run away from the ghosts. 20, 40 and $60 \mathrm{~mm} / \mathrm{s}$ might not be challenging for unimpaired users, seeing that they can come up with short path trajectories with less motion over different game runs. However, increasing speed to $100 \mathrm{~mm} / \mathrm{s}$, which is observed to be challenging even for unimpaired users, generally results in more motion.

3) Effect of map type: The different types of maps are designed according to the possible range of motion of slightly and moderately impaired patients for future use, and are expected to result in different strategies when played; Fig. 10 shows the cluster distributions with respect to these types. The blue cluster is more present in the Map Y since its small size makes it more difficult for the users to follow a strategy without being disturbed by the ghost(s).

In the Map O, we can see that no clusters (except the blue one) seem to clearly arise contrary to the Map G (the pink and dark green clusters together represent more than $30 \%$ of the distribution) or to the Map Y (the light blue and the yellow cluster together represent around $25 \%$ of the distribution). This may be due to the complexity of the Map $\mathrm{O}$, that allows a higher number of possible strategies. This complexity can be explained in two ways: (1) Around the path crossings, there are 3 or 4 possible directions to follow, which increase the variety of paths compared to following only corridors or corners. These crossings also help ghosts to find shorter paths to reach the Pacman. Only Map O includes such crossings with 4 connections. (2) Initial ghost positions in Map $\mathrm{O}$ have shorter distance paths to the initial position of the Pacman compared to the other maps. In Map Y and G, ghosts have to turn around a wall after starting their motion horizontally, while in Map $\mathrm{O}$ they move vertically without having a barrier. 

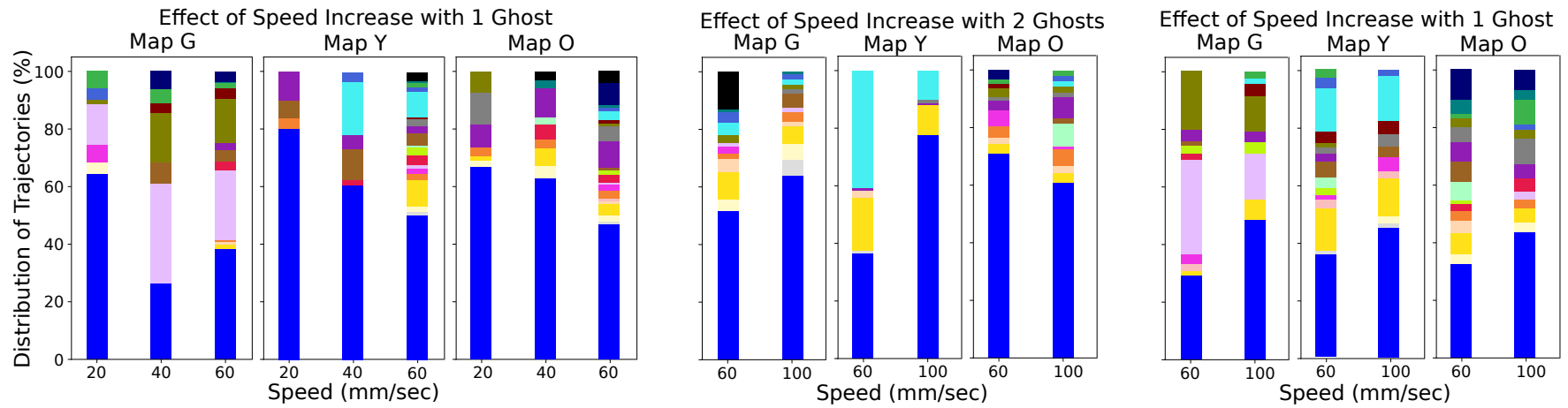

Fig. 9: Effect of ghost speed (20, 40,60, $100 \mathrm{~mm} / \mathrm{s})$ on trajectory distribution with changing number of ghosts.

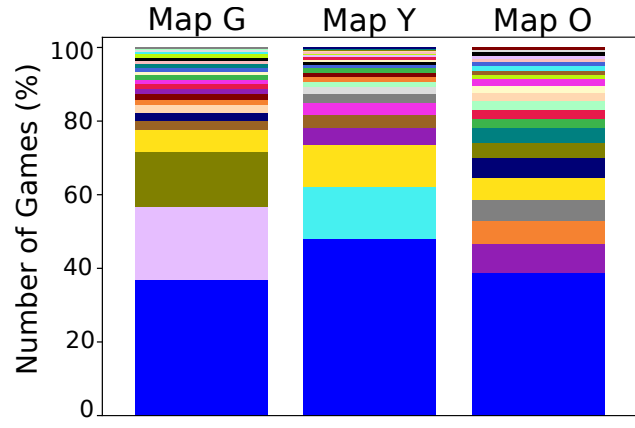

Fig. 10: Effect of map types on trajectory distribution.

\section{CONCLUSION AND DISCUSSIONS}

In the context of upper-arm home rehabilitation therapy, non-invasive and accurate metrics that can measure the effectiveness of rehabilitation can be crucial to adapt motor learning and motor rehabilitation to the individual patient. In this paper, we showed that the analysis of end-effector motion trajectories allow to observe performance differences and in particular the effect of game elements on motion performance.

Trajectories in this game can reflect both cognitive and motor abilities. A sudden trajectory change could be planned as part of a player's strategy to avoid the chasing ghost, or be attributed to motor limitations. This work was done as a first step towards building a data-driven cognitive and motor model of the players. This model would then be used to adapt the difficulty of the cognitive aspect (to keep the game challenging and engaging) and the motor aspect (to make the patient work on the specific motion that the therapist recommends). As part of this adaptation, we described several game elements, such as the number of ghost and their speed that can be tuned to reinforce the use of certain types of trajectory. This work will be improved by defining gesture matches between expected motion types and trajectories to provide more precise adaptation for expected exercise activities.

The main limitation of this study is that it was conducted with unimpaired participants rather than patients with neurological injuries, which may limit the conclusions for patients and have to be evaluated in patient studies. Therefore, as a future study, we will verify these baseline results with impaired patients in need of upper limb motor rehabilitation.
Our longer term plans include designing a real-time difficulty adaptation algorithm with trajectory expectation and evaluating it with participants with neurological injuries.

\section{REFERENCES}

[1] James H Cauraugh and Jeffery J Summers. Neural plasticity and bilateral movements: a rehabilitation approach for chronic stroke. Progress in neurobiology, 75(5):309-320, 2005.

[2] Roberto Colombo, Fabrizio Pisano, Alessandra Mazzone, Carmen Delconte, Silvestro Micera, M Chiara Carrozza, Paolo Dario, and Giuseppe Minuco. Design strategies to improve patient motivation during robot-aided rehabilitation. Journal of neuroengineering and rehabilitation, 4(1):3, 2007.

[3] Sharon M Nijenhuis, Gerdienke B Prange, Farshid Amirabdollahian, Patrizio Sale, Francesco Infarinato, Nasrin Nasr, Gail Mountain, Hermie J Hermens, Arno HA Stienen, Jaap H Buurke, et al. Feasibility study into self-administered training at home using an arm and hand device with motivational gaming environment in chronic stroke. Journal of neuroengineering and rehabilitation, 12(1):89, 2015.

[4] Maja Goršič, Minh Ha Tran, and Domen Novak. Cooperative cooking: A novel virtual environment for upper limb rehabilitation. In 2018 40th Annual International Conference of the IEEE Engineering in Medicine and Biology Society (EMBC), pages 3602-3605. IEEE, 2018.

[5] C Bryanton, J Bosse, Marie Brien, Jennifer Mclean, Anna McCormick, and Heidi Sveistrup. Feasibility, motivation, and selective motor control: virtual reality compared to conventional home exercise in children with cerebral palsy. Cyberpsychology \& behavior, 9(2):123128, 2006.

[6] Bruno Bonnechère, Bart Jansen, Lubos Omelina, Jan Van Sint, et al The use of commercial video games in rehabilitation: a systematic review. International journal of rehabilitation research, 39(4):277290, 2016.

[7] Lesley Axelrod, Geraldine Fitzpatrick, Jane Burridge, Sue Mawson, Penny Smith, Tom Rodden, and Ian Ricketts. The reality of homes fit for heroes: design challenges for rehabilitation technology at home. Journal of Assistive Technologies, 3(2):35-43, 2009.

[8] Arzu Guneysu Ozgur, Maximilian J. Wessel, Wafa Johal, Kshitij Sharma, Ayberk Özgür, Philippe Vuadens, Francesco Mondada, Friedhelm C. Hummel, and Pierre Dillenbourg. Iterative design of an upper limb rehabilitation game with tangible robots. In Proceedings of the 2018 ACM/IEEE International Conference on Human-Robot Interaction, pages 241-250. ACM, 2018.

[9] Philipp J Koch and Friedhelm C Hummel. Toward precision medicine: tailoring interventional strategies based on noninvasive brain stimulation for motor recovery after stroke. Current opinion in neurology, 30(4):388-397, 2017

[10] Arzu Guneysu, Recep D. Siyli, and Albert A. Salah. Auto-evaluation of motion imitation in a child-robot imitation game for upper arm rehabilitation. In Robot and Human Interactive Communication, 2014 RO-MAN: The 23rd IEEE International Symposium on, pages 199204. IEEE, 2014.

[11] Jae-Gil Lee, Jiawei Han, Xiaolei Li, and Hector Gonzalez. Traclass: trajectory classification using hierarchical region-based and trajectorybased clustering. Proceedings of the VLDB Endowment, 1(1):10811094, 2008.

[12] D Dimitrescu. Hierarchical pattern classification. Fuzzy Sets and Systems, 28(2):145-162, 1988. 